\title{
Design of an Ontology for Decision Support in VR Exposure Therapy
}

\author{
Joris Heyse \\ Ghent University, IDLab, Department \\ of Information Technology - imec \\ Ghent, Belgium \\ joris.heyse@ugent.be \\ Anissa All \\ Ghent University, MICT, Department \\ of Communication Sciences - imec \\ Ghent, Belgium \\ anissa.all@ugent.be
}

\author{
Femke Ongenae \\ Ghent University, IDLab, Department \\ of Information Technology - imec \\ Ghent, Belgium \\ femke.ongenae@ugent.be \\ Femke De Backere \\ Ghent University, IDLab, Department \\ of Information Technology - imec \\ Ghent, Belgium \\ femke.debackere@ugent.be
}

\author{
Jolien De Letter \\ Ghent University, MICT, Department \\ of Communication Sciences - imec \\ Ghent, Belgium \\ jolien.deletter@ugent.be \\ Filip De Turck \\ Ghent University, IDLab, Department \\ of Information Technology - imec \\ Ghent, Belgium \\ filip.deturck@ugent.be
}

\begin{abstract}
Virtual Reality (VR) is finding its way into many domains, including healthcare. Therapists greatly benefit from having any scenario in VR at their disposal for exposure therapy. However, adapting the VR environment to the needs of the patient is time-consuming. Therefore, an intelligent decision support system that takes context information into account would be a big improvement for personalised VR therapy. In this paper, a semantic ontology is presented for modelling relevant concepts and relations in the context of anxiety therapy in VR. The necessary knowledge was collected through workshops with therapists, this resulted in a layered ontology. Furthermore, semantic reasoning through logical rules enables deduction of interesting high-level knowledge from low-level data. The presented ontology is a starting point for further research on intelligent adaptation algorithms for personalised VR exposure therapy.
\end{abstract}

\section{CCS CONCEPTS}

- Theory of computation $\rightarrow$ Semantics and reasoning; • Information systems $\rightarrow$ Expert systems; • General and reference $\rightarrow$ Design.

\section{KEYWORDS}

Ontology design, Decision support, eHealth, VRET

\section{INTRODUCTION}

In recent years, an uptake of Virtual Reality (VR) has been seen in the healthcare domain, specifically in cognitive behaviour therapy (CBT) [13]. VR allows to submerge the patient in an immersive Virtual Environment (VE) in which effective therapy can take place.

Permission to make digital or hard copies of all or part of this work for personal or classroom use is granted without fee provided that copies are not made or distributed for profit or commercial advantage and that copies bear this notice and the full citation on the first page. Copyrights for components of this work owned by others than the author(s) must be honored. Abstracting with credit is permitted. To copy otherwise, or republish, to post on servers or to redistribute to lists, requires prior specific permission and/or a fee. Request permissions from permissions@acm.org.

PervasiveHealth 2019, May 20-23, 2019, Trento, Italy

(c) 2019 Copyright held by the owner/author(s). Publication rights licensed to ACM

ACM ISBN 978-x-xxxx-xxxx-x/YY/MM...\$15.00

https://doi.org/10.1145/nnnnnnn.nnnnnnn
The biggest advantage of VR in therapy is that the environment can resemble any imaginable scenario. This is especially interesting for VR Exposure Therapy (VRET), in which the patient is exposed to a specific scenario that elicits fear. Effective therapy, requires that the $\mathrm{VE}$ is configured and adapted as precisely as possible. However, this is not an easy task, manual configuration of the VE trough dozens of parameters is both time-consuming and cumbersome. Therefore, a therapist would benefit from a system that assists with this task.

Decision support has been introduced in healthcare in the late sixties and it has evolved ever since [7]. Decision support systems (DSSs) nowadays, are characterised by vast amounts of heterogeneous data from various physical devices, such as wearables, smart watches and sensors, and written documents, such as notes, logging documents and questionnaires. After processing, this data can provide valuable input for the treatment of patients. It is important to bear in mind that the purpose of a DSS is to support doctors and healthcare practitioners, the domain experts need to have the last say in making the decisions. Meaning, a DSS should only play a supportive role by making suggestions to the domain experts, it should not make decisions itself [2].

A DSS consists of a model that consolidate data from heterogeneous sources for easy processing and utilisation. Additionally, some logic is applied on this data model to extract and infer additional knowledge for decision making. Semantic Web technologies offer excellent tools for these purposes [9]. Semantic ontologies are used to model relevant domain knowledge, it models the concepts and defines relationships between these concepts in an unambiguous way [9]. Additionally, semantic reasoners are able to unveil hidden, high-level, knowledge from low-level data in an ontology [11]. By defining logical rules, new interesting knowledge can be inferred from the data in the model. Designing an ontology is a complex task, as it should model the required knowledge for the given application in enough detail. Many ontologies already exist for many different domains in healthcare applications. However, no ontology has been designed yet for the specific purpose of VRET.

Within the PATRONUS project ${ }^{1}$, a VRET application is being designed, where physiological data is taken into account to offer truly personalised Exposure Therapy (ET) [5]. The VE is adapted

\footnotetext{
${ }^{1}$ https://www.imec-int.com/en/what-we-offer/research-portfolio/patronus
} 
to suit each individual case. A model that takes into account all necessary context and background information will help the therapist in adjusting the VE. The aim of the project is to design a novel application, as currently no solution exists, that allows intelligent adaptations to the VE.

This paper presents a semantic ontology, specifically for ET in VR. The resulting ontology will be part of a DSS that aids therapists in configuring VE to the specific needs of a patient. The DSS is developed in context of the PATRONUS project [5]. The remainder of this paper gives an overview of existing tools for VRET and discusses some related ontologies in Section 2. Following that, in Section 3 the design methodology is explained, and the proposed semantic ontology is presented in Section 4. The paper finishes with a discussion and a highlight of the conclusions.

\section{RELATED WORK}

A few tools already exist for VRET such as Psious ${ }^{2}$ and $\mathrm{CleVR}^{3}$. These platforms require the VE to be manually configured with many parameters, through a dashboard, by the therapist. Currently, no tools exist that also incorporate biofeedback from sensors for objective anxiety analysis. Although Psious does allow for connecting a sensor and monitoring physiological parameters, the assessment still has to be performed manually by the therapist. Consequently, none of the current solutions includes a system for autonomous suggestions for adaptation of the VE based on anxiety levels or any other context information.

One of the main pillars of the Semantic Web, is reuse of knowledge. Specifically, this means that ontologies should be reusable. Already many ontologies exist for healthcare domains, covering many levels of granularity. Semantic Web pleads for reusing and combining the existing ontologies as much as possible. A search of the current literature revealed some related ontologies in the area of CBT and mental disorder.

The Mental Health Ontology [3] and the Ontology of Mental Disease (OMD) [1] are ontologies that provide a classification of many mental health disorders and links them to possible treatments. OMD is developed specifically as a clear and unambiguous document to communicate about mental diseases. It gives definitions for the different categories of diseases and also describes symptoms. These definitions are described at different levels of granularity. The Emotion Ontology (EMO) [4], which builds further on OMD, models concepts related to emotions. As for mental diseases, there is also a lot of ambiguity and confusion when talking about emotions. EMO tries to solve this confusion by classifying emotions and stating clear definitions. EMO also links the emotion entities to the terminology in OMD which allows them to work great together. B. Hu et al., present an ontology for modelling context for mental disorder patients. The ontology allows to record logs, statistics and treatments, and query them [6]. Similarly, I. Zaragozá et al., presents an ontology for modelling patient context in a mental health application for obesity treatment [12].

Some of these ontologies $[1,3,4]$ provide a classification system for concepts in the mental health domain. They could be useful for situating VRET treatment and some of it concepts in the broad

\footnotetext{
${ }^{2}$ www.psious.com

${ }^{3}$ www.clevr.net
}

landscape of all treatments, which are outside of the scope of this paper. However, they do not model the interaction between the relevant concepts for VRET, and are therefore not reusable in our work. Alternatively, some other ontologies $[6,12]$ are very detailed and application specific. Therefore their reusability is also minimal for other applications such as VRET.

The initial search of the literature revealed no ontologies for exposure therapy or VRET. A new ontology has to be designed to fill this gap in the literature.

\section{DESIGN METHODOLOGY}

There exist many methodologies for designing ontologies. Using a combination of multiple techniques is also possible. In our work, primarily the methodology presented by F. Ongenae et al. is applied [8]. The authors state that their method for ontology design is an iterative process that requires close interaction with domain experts and (semantic) engineers. The domain experts provide the knowledge to be modelled as well as provide feedback over multiple iterations. The engineers model that knowledge using the semantic technologies and implement it into a domain application for evaluation with end users. The proposed methodology is based on workshops with domain experts for extracting the relevant knowledge. The output of the workshops are a set of concepts and relations that are used for constructing the ontology.

Two workshops were held, one with 4 and one with 3 therapists. Having multiple workshops prevents that the output is biased towards one opinion. During the workshops, a moderator asks questions to the therapist to help them focus on interesting problems and steer them away from non-relevant problems. At the same time, a semantic engineer is writing down, on large sheets of paper, the prominent concepts that play an important role in therapy. After the workshops, the collected knowledge was extended with knowledge from written documents that therapists currently already use for in vivo ET, such as questionnaires and logging documents. These provide further insights into which questions therapists usually ask the patient, as well as which information they are interested in.

Defining the scope of an ontology is important. An ontology that is too large and complex will have computational implications as well as reduce the comprehensibility. However, a too small ontology could lack the necessary knowledge. In this work, the scope of the ontology was defined by composing a list of competence questions, i.e., a list of questions for which the ontology should contain the necessary knowledge to answer them.

\section{RESULTS}

The outcome of the workshops are unstructured and still need interpretation, they reveal which knowledge is most important according to the therapist. Next, the knowledge is put into a structured ontology. Finally, some knowledge is more complex than the relations between concepts, logical rules are employed for adding knowledge to the ontology.

\subsection{Workshop outcomes}

On a high-level, the workshops revealed that a patient typically has different responses on different stimuli. The therapist then focusses on testing which stimulus elicits which response. The stimuli can 
be anything, e.g., objects, sounds or thoughts. A response is in fact a very complicated concept, it can have many complex underlying reasons. The interpretation of the behaviour of the patient is left for the therapist, but the high-level response should be modelled in the ontology. The role and meaning of an objective measure for anxiety is a topic of discussion among therapists. It remains to be researched to which extend objective anxiety measures are relevant for decisions about VE adaptations. Therefore this objective response has to be modelled as well. In practice, therapists currently employ subjective metrics for anxiety levels [10]. These do play an important role in the decision making process during therapy.

Therapists expressed which information and knowledge is important for making decisions about which therapy exercise to give. For defining the scope of our VRET ontology, the following nonexhaustive list of questions is constructed based on the input from the therapists. That is, our ontology should at least be able to support answers for the following questions:

- Which stimuli are known to elicit anxiety for a patient?

- Which response is expected for a certain stimulus?

- Which stimuli have not been tested during previous exercises?

- Does a stimulus affect the subjective anxiety metric?

- Which stimuli where most effective?

- What where the objective anxiety levels for all exercises?

\subsection{Layered Ontology}

The proposed ontology, presented in Figure 1, consists of three layers. A simple, and general upper-ontology, and specialised ontologies for ET and VRET. The upper-ontology models general concepts relevant for CBT without referring to ET or VR related concepts specifically. The ET ontology relates the upper-ontology to ET specific concepts. Subsequently, the VRET relates those concepts from ET to the domain of VRET.

On the highest level, shown in the upper left of Figure 1, there are Patient, Hypothesis, Stimulus, Response and Exercise. Each person can have multiple Hypothesis about their Response to a certain Stimulus. The hypothesis is an expectation of how a patient will respond to a set of stimuli. Once a hypothesis is tested, there is an observation that either confirms or rejects the hypothesis. Therefore, each hypothesis should be tested with some Exercise. An Exercise tests a single Hypothesis and is performed by a Patient.

Specifically for ET, the hypotheses are expressed in terms of stimuli that occur in real life (in vivo) and can be separated into Events and characteristics of the environment (EnvironmentProperty). Responses, are part of a more complex classification. As seen in the upper right of Figure 1, there are Subjective and Objective responses. The subjective response are either perceived by the therapist or the patient itself, while the objective response is calculated from data collected through wearables. The commonly used, Subjective Unit of Disturbance (SUD) score [10], is a subjective score for anxiety provided by the patient. Behaviour, is the most complex concept, in our ontology it is simplified to be either observations of Anxiety Behaviour, Avoidance Behaviour or Dependancy Behaviour. I.e., a patient can behave anxiously because of stimuli, they can avoid it because or a patient feels safe because of stimuli.

VRET is a tool for executing exercises. In our paradigm, an exercise is a simulated way of testing an in vivo hypothesis. Thus, the stimuli and hypotheses should have an equivalent in VR. Specifically, a stimulus in VR is a simulation of what that stimulus looks or feels like in real life. Therefore, a relation exists that links in vivo stimuli and VR stimuli, indicating their equivalence. A hypothesis can be considered as a scenario in VR. A scenario in turn, consists of a VR environment and VR events. E.g., an environment that consists of an elevator, or another one that is taking place in a car. Finally, in VR we differentiate between VR exercises at the therapist office and VR exercises at home. This differentiation is necessary because of the envision application described in the PATRONUS project [5], which focusses on VR exercises at the therapists office on the one hand, and homework VR exercises on the other hand.

\subsection{Ontology Reasoning}

By using rules to reason over the ontology, new knowledge can be discovered. Description logic (DL), a subset of first order logic, allows to formulate such rules. Two kinds of reasoning are commonly used, ontology reasoning and user-defined reasoning [11]. Ontology reasoning allows to reason about hierarchical properties of classes and properties. It also allows reasoning for concept satisfiability, class subsumptions and ontology inconsistencies [11]. User-defined reasoning allows to deduce high-level connections from lower-level data. This is particular interesting because it allows to include use-case dependent logic into the knowledge base. Applied on our use case, rules can be formulated for the following examples.

- If a patient assigns a SUD score above a certain threshold, then they experience anxiety behaviour: hasTestedResponse(?h, ?r) $\sqcap S U D(? r) \sqcap$ hasSUDScore(?r, ?v) $\sqcap$ is GreaterThan(?v, 6) $\Longrightarrow$ hasTestedResponse(?h, AnxietyBehaviour)

- A VR scenario testing a hypothesis should at least include the stimuli of the hypothesis: Event(?s1) $\sqcap$ VREvent(?s2) $\sqcap$ hasVREquivalent(?s1, ?s2) $\sqcap$ testsStimulus(?h, ?s1) $\sqcap$ VRScenario(?h) $\Longrightarrow$ triggersEvent(?h, ?s2)

\section{DISCUSSION}

In this paper, a first iteration of an ontology for VRET is presented. The workshops with the therapists made clear that CBT is quite complex and hard to structure in simple rules and relations. Regardless, a model, as simple as possible for our specific application is designed. Future iterations will reveal if the level of complexity of this ontology with its rules is high enough for practical application. Important to keep in mind is that the model with an accompanying algorithm should only support the therapist in making decisions, and not replace the therapist entirely.

\section{CONCLUSION}

During this research phase, the first steps towards an ontology for a DSS in VRET are taken. Therapists were consulted to extract the knowledge that is needed for decision making. It is clear that CBT is complex, and that clear rules or guidelines not always exist. The presented ontology consists of three layers, one upper-ontology with general concepts for therapy, one on ET specifically, and one that applies ET in VR. This work is a starting point for future iterations of this ontology and provides a data model for future 


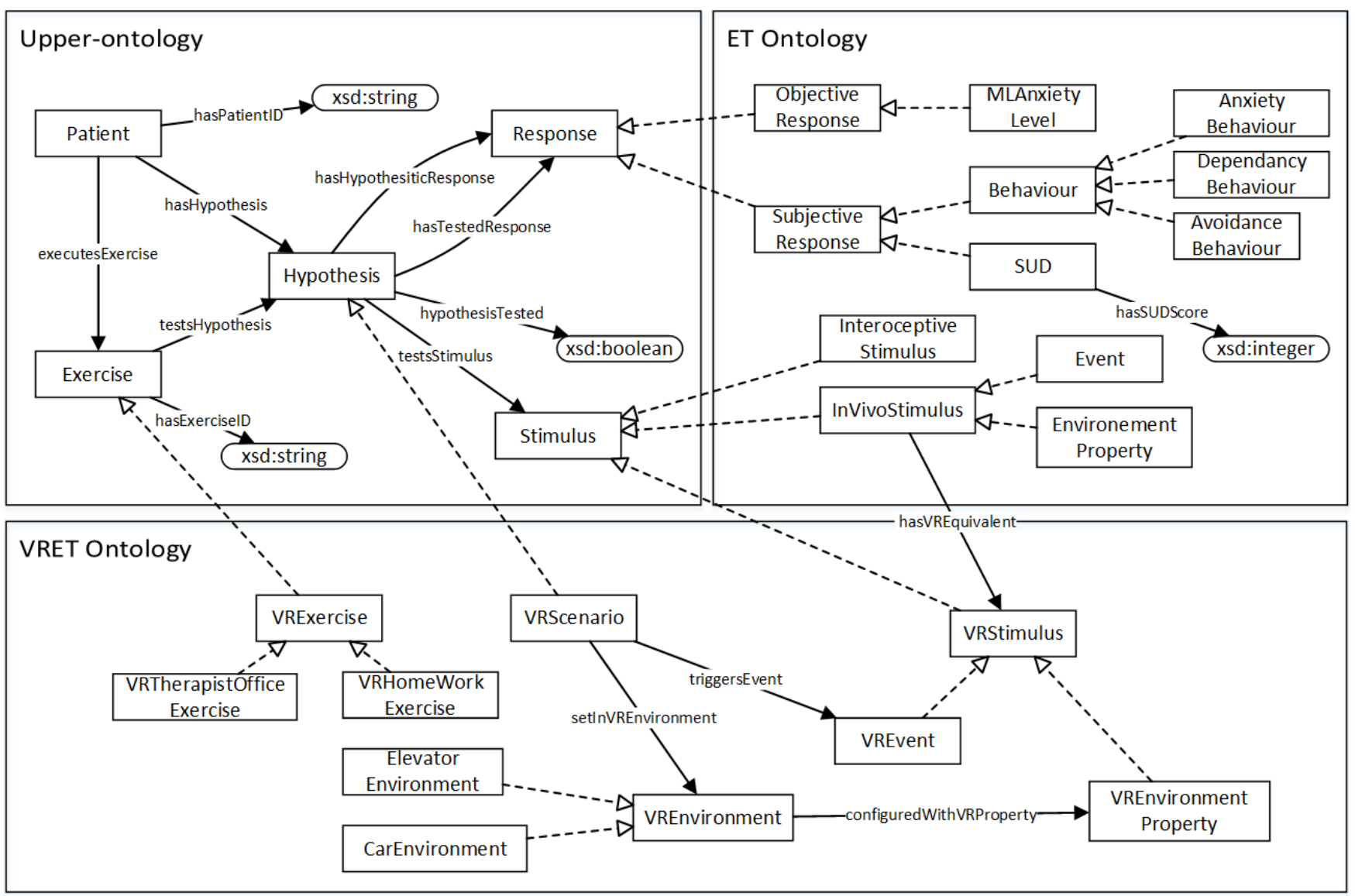

Figure 1: Diagram of the concepts in the ET and VRET ontologies related to the upper-ontology, and their properties. The arrows with dashed lines indicate subclass relationships. The arrows with a full lines represent properties of classes.

adaptation algorithms for VRET. Implementation in a testing setup will provide further input for improvements to shortcomings of this model.

\section{ACKNOWLEDGMENTS}

This research is funded by the imec.icon project PATRONUS, which was co-financed by imec and VLAIO, it brings together the following partners: The Human Link, PreviewLabs, Bazookas, Van Roey Automation, Ghent University - IDLab, Ghent University - MICT and imec - WHS.

\section{REFERENCES}

[1] Werner Ceusters and Barry Smith. 2010. Foundations for a realist ontology of mental disease. Fournal of Biomedical Semantics 1, 1 (2010).

[2] Kathrin Cresswell, Azeem Majeed, David W Bates, and Aziz Sheikh. 2013. Computerised decision support systems for healthcare professionals: an interpretative review. Journal of Innovation in Health Informatics 20, 2 (2013), 115-128.

[3] M. Hadzic, M. Chen, and T. S. Dillon. 2008. Towards the Mental Health Ontology. In 2008 IEEE International Conference on Bioinformatics and Biomedicine. IEEE, 284-288.

[4] Janna Hastings, Werner Ceusters, Barry Smith, and Kevin Mulligan. 2011. Dispositions and Processes in the Emotion Ontology. In Proceedings of the 2nd International Conference on Biomedical Ontology. CEUR Workshop Proceedings, 71-78.
[5] Joris Heyse, Femke Ongenae, Femke De Backere, and Filip De Turck. 2018. Personalisation of Exercises in VRET. In Workshops at the Thirty-Second AAAI Conference on Artificial Intelligence. AAAI.

[6] Bin Hu, Bo Hu, Jizheng Wan, Majoe Dennis, Hsiao-Hwa Chen, Lian Li, and Qingguo Zhou. 2010. Ontology-based ubiquitous monitoring and treatment against depression. Wireless Communications and Mobile Computing 10, 10 (2010), 1303-1319.

[7] David P McCallie. 2016. Clinical Decision Support: History and Basic Concepts. In Healthcare Information Management Systems. Springer, 3-19.

[8] Femke Ongenae, Pieter Duysburgh, Nicky Sulmon, Mathijs Verstraete, Lizzy Bleumers, Saar De Zutter, Stijn Verstichel, Ann Ackaert, An Jacobs, and Filip De Turck. 2014. An ontology co-design method for the co-creation of a continuous care ontology. Applied Ontology 9, 1 (2014), 27-64.

[9] Nirmalya Roy, Tao Gu, and Sajal K. Das. 2010. Supporting pervasive computing applications with active context fusion and semantic context delivery. Pervasive and Mobile Computing 6, 1 (2010), 21-42.

[10] Joseph Wolpe. 1990. The practice of behavior therapy. Pergamon Press.

[11] Xiao Hang Wang, Da Qing Zhang, Tao Gu, and Hung Keng Pung. 2004. Ontology based context modeling and reasoning using OWL. In IEEE Annual Conference on Pervasive Computing and Communications Workshops, 2004. Proceedings of the Second. IEEE, 18-22.

[12] Irene Zaragozá, Jaime Guixeres, and Mariano Alcañiz. 2009. Ontologies for intelligent e-therapy: application to obesity. In International Work-Conference on Artificial Neural Networks. Springer, 894-901.

[13] Heidi M. Zinzow, Johnell O. Brooks, Patrick J. Rosopa, Stephanie Jeffirs, Casey Jenkins, Julia Seeanner, Alyssa McKeeman, and Larry F. Hodges. 2018. Virtual Reality and Cognitive-Behavioral Therapy for Driving Anxiety and Aggression in Veterans: A Pilot Study. Cognitive and Behavioral Practice 25, 2 (2018), 296-309. 